\title{
1 pOPIN-GG: A resource for modular assembly in protein expression
}

\section{vectors}

3

4 Adam R. Bentham ${ }^{1, *}$, Mark Youles ${ }^{2, *}$, Melanie N. Mendel ${ }^{1,3}$, Freya A. Varden ${ }^{1}$, Juan Carlos De

5 la Concepcion ${ }^{1,4}$, Mark J. Banfield ${ }^{1}$

$6{ }^{1}$ Department of Biochemistry and Metabolism, John Innes Centre, Norwich Research Park,

$7 \quad$ Norwich, NR4 7UH, UK

$8{ }^{2}$ The Sainsbury Laboratory, University of East Anglia, Norwich Research Park, NR4 7UH,

9 Norwich, UK

$10{ }^{3}$ Current address: Plant-Microbe Interactions, Utrecht University, Padualaan 8, Utrecht, $113584 \mathrm{CH}$, Netherlands

$12{ }^{4}$ Current address: Gregor Mendel Institute of Molecular Plant Biology, Austrian Academy of

13 Sciences, Vienna, 1030, Austria

14 Corresponding author email: adam.bentham@jic.ac.uk, mark.banfield@jic.ac.uk

$15{ }^{*}$ Contributed equally to this work.

17 ORCIDs:

18 Adam R. Bentham: 0000-0001-5906-0962

19 Melanie N. Mendel: 0000-0003-2409-7479

20 Freya A. Varden: 0000-0003-4224-2326

21 Juan Carlos De la Concepcion: 0000-0002-7642-8375

22 Mark J. Banfield: 0000-0001-8921-3835 


\section{Highlights}

\section{Keywords}

34 Modular cloning, Golden Gate, high-throughput protein expression, solubility tags, copurification

36

37 Word Count: 3,448 (Abstract, Introduction, Results, Discussion, Experimental Procedures,
- pOPIN-GG expression vectors allow for modular cloning enabling rapid screening of purification and solubility tags at no loss of expression compared to previous vectors.

- Cloning into the pOPIN-GG vectors can be performed from PCR products or from level 0 vectors containing the required parts.

- Several vectors with different resistances and origins of replication have been generated allowing the effective co-expression and purification of protein complexes.

- All pOPIN-GG vectors generated here are available on Addgene, as well as level 0 acceptors and tags. 


\section{Abstract}

40 The ability to recombinantly produce target proteins is essential to many biochemical,

41 structural, and biophysical assays that allow for interrogation of molecular mechanisms behind

42 protein function. Purification and solubility tags are routinely used to maximise the yield and

43 ease of protein expression and purification from E. coli. A major hurdle in high-throughput

44 protein expression trials is the cloning required to produce multiple constructs with different solubility tags. Here we report a modification of the well-established pOPIN expression vector suite to be compatible with modular cloning via Type IIS restriction enzymes. This allows users

47 to rapidly generate multiple constructs with any desired tag, introducing modularity in the 48 system and delivering compatibility with other modular cloning vector systems, for example streamlining the process of moving between expression hosts. We demonstrate these constructs maintain the expression capability of the original pOPIN vector suite and can also be used to efficiently express and purify protein complexes, making these vectors an excellent resource for high-throughput protein expression trials. 
54 Understanding protein function is key to answering many biological questions. Biochemical, structural, and biophysical techniques that probe the molecular mechanisms behind protein function are reliant on the production of purified protein for use in these assays. Procedures for protein expression and purification from Escherichia coli have been advanced by methodologies which allow for the high-throughput generation of constructs (Rosano and Ceccarelli, 2014). Intrinsic to generating soluble protein of the more difficult targets in E. coli is the capacity to test multiple solubility tags, such as the Small Ubiquitin-like modifier (SUMO) or the Maltose Binding Protein (MBP) tags, which allow for the production of proteins that would be otherwise insoluble (di Guana et al., 1988; Malakhov et al., 2004). Further, the use of purification tags frequently allows for rapid capture of proteins of interest from cell lysates. However, purification and solubility tags are often vector-linked, encoded in the expression vector upstream of the cloning site of the target gene. As such, the lack of modularity of purification and solubility tags in expression vectors presents a bottleneck in high-throughput expression screens as the user is limited to the solubility tags encoded in the vectors available to them. Therefore, tackling the problem of modularity represents an opportunity to increase the efficiency of expression trials, and readily allows for the incorporation of novel purification and solubility tags as they are developed.

The pOPIN vector suite, generated by the Oxford Protein Production Facility (OPPF), is a set of expression vectors encoding various purification and solubility tags at either the $\mathrm{N}$ - or $\mathrm{C}$ terminus of the gene of interest (GOI) (Berrow et al., 2007). These vectors allow for a straightforward cloning method via ligation independent cloning (LIC) and rapid generation of constructs. Furthermore, the pOPIN vectors are compatible with multiple hosts, with many being able to be used in bacterial, insect and mammalian cell hosts (Berrow et al., 2007). One shortcoming of these excellent vectors is a lack of modularity, meaning users are restricted to the solubility tags provided in the vector suite. 
81 Golden Gate cloning (also known as Greengate cloning or MoClo) represents a fast and 82 efficient method of cloning genes through the use of Type IIS restriction endonucleases that cut outside their recognition site to reveal user defined four nucleotide overhangs at both the 5' and 3' ends of the DNA (Engler et al., 2008). These overhangs can be exploited to allow scarless cloning, as well as for design and assembly of multiple DNA fragments in a single ligation reaction (Padgett and Sorge, 1996). They also allow for efficient subcloning between vectors (Engler et al., 2008). Golden Gate cloning allows for the generation of diverse "level 0" parts, which can be promoters, the GOI, terminators, epitope tags etc. These can then be assembled into "level 1" expression cassettes, which themselves can be further cloned along with other level 1 expression cassettes to give rise to "level 2" multi-gene assemblies. Moreover, the scarless nature of Golden Gate cloning makes the technique excellent for synthetic design approaches such as assembling chimeric proteins, allowing the generation of protein domain-swaps, or tagging proteins. Due to its high efficiency, modularity, and well established sequential cloning strategy, Golden Gate cloning has been incorporated in multiple vector systems for eukaryotic expression, such as plants or insect cells (Engler et al., 2014, 2009; Neuhold et al., 2020).

Here, we present a modified pOPIN vector suite we call pOPIN-GG that takes advantage of Golden Gate cloning (Engler et al., 2009, 2008) to incorporate modularity into the pOPIN vectors without disrupting efficacy of expression. These vectors also allow cross-compatibility with other Golden Gate systems, such as plant expression binary vectors (Engler et al., 2014; Patron et al., 2015), and simple one-pot reactions which allow for the bespoke construction of expression vectors containing the $\mathrm{GOI}$ and desired purification or solubility tags. We demonstrate that the pOPIN-GG vectors express comparable levels of protein to those of the classic pOPIN suite, whilst also enabling the user to selectively vary the choice of tags. This incorporation of modularity in protein expression supplied by POPIN-GG vectors further 
bioRxiv preprint doi: https://doi.org/10.1101/2021.08.10.455798; this version posted August 10,2021 . The copyright holder for this preprint

(which was not certified by peer review) is the author/funder, who has granted bioRxiv a license to display the preprint in perpetuity. It is made available under aCC-BY 4.0 International license.

107 advances high-throughput protein expression trials and subsequent preparative purification in

108 E. coli. 
110 Cloning into the $p O P I N-G G$ vectors.

111 Cloning into the pOPIN-GG vectors follows standard Golden Gate protocols where matching

112 overhangs between acceptor vector and inserts, revealed by digestion with a Type IIS 113 endonuclease, Bsal, are ligated with T4 ligase (Engler et al., 2009, 2008). Here, we have 114 engineered two sets of overhangs into the pOPIN-GG vectors: 5' CCAT and 3' GCTT for 115 pOPIN-GG N-terminal tag compatible vectors (pPGN), and 5' AATG and 3' GCTT overhangs 116 for pOPIN-GG C-terminal tag compatible vectors (pPGC). The pPGN and pPGC vectors have 117 been developed with two different options for antibiotic selection, carbenicillin (the pPGN-C 118 and pPGC-C vectors) and kanamycin (the pPGN-K and pPGC-K vectors), which allows for 119 co-expression via bacterial co-transformation. Annotated vector maps of these pOPIN-GG 120 acceptors are shown in Figure 1, detailing the changes made to the original pOPIN-F and 121 pOPIN-A vectors (the modifications are further described in the experimental procedures).

122 Cloning of GOls into the pOPIN-GG vectors are based on a common syntax for Type IIS endonuclease-mediated assembly (Patron et al., 2015). For cloning of N-terminally tagged

124 GOls into pPGN, the desired N-terminal tag must have the overhangs 5' CCAT and 3' AATG, and the GOI have the overhangs 5' AATG and 3' GCTT, when revealed by digestion with Bsal.

126 For cloning into PPGC, the GOI requires the overhangs 5' AATG and 3' TTCG and the C-

127 terminal tag must have the overhangs 5' TTCG and 3' GCTT. In addition, a GOI encoding an 128 untagged protein can be cloned by using the 5' AATG and 3' GCTT overhangs and one of the 129 C-terminal tagging compatible pPGC vectors, which contain the 5'AATG and 3' GCTT 130 overhangs. Figure 2 visualises the compatible overhangs between vector, tag and insert for 131 cloning to produce $\mathrm{N}$-terminally tagged, C-terminally tagged, and untagged proteins.

132 Overhangs can be introduced into a desired sequence through either PCR with primers 133 containing overhangs and a Bsal site (Table 1), or through Bsal-digestion of a compatible level 1340 entry vector containing either tag or insert of interest. This highlights a major advantage of 135 the pOPIN-GG vectors, by which a single PCR amplification or level 0 vector containing the 
136 GOI with the appropriate N-terminal or C-terminal tagging overhangs can be used for multiple

137 simultaneous digestion-ligation reactions as the overhangs remain universal between

138 acceptor, tag, and insert. pOPIN-GG acceptors along with multiple level 0 vectors containing

$139 \mathrm{~N}$-terminal and C-terminal tags with the necessary overhangs for cloning are listed in Table 2.

141 The pOPIN-GG vectors maintain protein expression levels comparable to the original pOPIN

142 vectors.

143 To test the efficacy of the pOPIN-GG vectors for protein expression, we cloned AVR-PikF (a

144 small, secreted effector protein from the blast fungus Magnaporthe oryzae (Longya et al., 145 2019)), into the vectors pOPIN-F (N-terminal 6xHIS-3C), pOPIN-S3C (N-terminal 6xHIS146 SUMO-3C), pOPIN-M (N-terminal 6xHIS-MBP-3C) and pOPIN-E (C-terminal 6xHIS 147 uncleavable), and generated the equivalent constructs using our pOPIN-GG system (see experimental procedures). We also cloned AVR-PikF with a 6xHIS-GB1 solubility tag

149 (Kobashigawa et al., 2009), to which we did not have access to the pOPIN equivalent, to 150 demonstrate the adaptability of the pOPIN-GG system. Figure 3 shows a comparison between AVR-PikF expressed in the E. coli SHuffle strain using the gene cloned in the original pOPIN vectors and the pOPIN-GG vectors and after benchtop $\mathrm{Ni}^{2+}$-immobilised metal affinity chromatography (IMAC) purification. We observed no significant differences in the yield of protein between the pOPIN vectors and new pOPIN-GG vectors, demonstrating the pOPINGG vectors retain the same capacity for protein production as the parent vectors.

157 The pOPIN-GG vectors are compatible with co-expression.

158 To further demonstrate the flexibility of the pOPIN-GG vectors, we next cloned an interaction partner of AVR-PikF, OsHIPP19 (Maidment et al., 2021), into pPGN-C with a 6xHIS-GB1 tag. We co-transformed $E$. coli SHuffle cells with the 6xHIS-GB1-OsHIPP19 vector and an untagged variant of AVR-PikF (in pPGC-K), expressed the proteins, and performed $\mathrm{Ni}^{2+}-\mathrm{IMAC}$ coupled with size exclusion chromatography (SEC) to purify a complex between AVR-PikF and OsHIPP19 (Figure 4). SDS-PAGE shows both proteins are expressed and purified, with 
bioRxiv preprint doi: https://doi.org/10.1101/2021.08.10.455798; this version posted August 10, 2021. The copyright holder for this preprint

(which was not certified by peer review) is the author/funder, who has granted bioRxiv a license to display the preprint in perpetuity. It is made available under aCC-BY 4.0 International license.

164 AVR-PikF co-purified along with OsHIPP19 (Figure 4). These results demonstrate the pOPIN-

165 GG vectors can be used for efficient expression and purification of protein complexes. 


\section{Discussion}

167 The ability to test multiple purification and solubility tags in E. coli expression trials is important

168 for determining the best conditions for protein production. High-throughput expression trials

169 allow the user to explore potential avenues for successful protein production, and a key step

170 is the ability to clone the gene of interest easily and effectively into a variety of constructs with

171 different purification and solubility tags. Here we describe pOPIN-GG, a modification of the

172 pOPIN vector suite, which allows for modular cloning using Golden Gate. The redesigned

173 pOPIN-GG vector suite retains the expression levels of the pOPIN vectors (with the proteins

174 we tested) while enhancing their modularity. A major advantage of the pOPIN-GG system is it

175 allows for the rapid introduction new purification and solubility tags as they emerge, in a

176 straightforward and easy to clone manner. The different pOPIN-GG acceptors are cross-

177 compatible for co-expression, allowing for ease of co-expression and purification of protein

178 complexes. Finally, the utility of the pOPIN-GG vectors presented here have already been

179 successfully tested by the community for the expression and purification of a fungal effector

180 from Parastagonospora nodorum (Outram et al., 2021), as well as for detailing the structural

181 mechanisms underpinning the evolution of Magnaporthe oryzae effectors for a specific host

182 target (Bentham et al., 2021). 


\section{Conclusions}

184 The pOPIN vector suite is a well-established and effective $E$. coli vector system for protein

185 expression and purification. By modifying the pOPIN vectors to be Golden Gate compatible

186 we have introduced modularity to the system, providing the user with the advantages of

187 modular cloning and allowing the incorporation new purification and solubility tags to the vector

188 repertoire, streamlining the vector assembly step, with no cost to the expression efficacy of

189 the system. We have made the pOPIN-GG vectors available on Addgene

190 (https://www.addgene.org/Mark_Banfield) as a package with level 0 and level 1 acceptors,

191 and level $0 \mathrm{~N}$-terminal and C-terminal tag constructs. 


\section{Experimental Procedures}

193 Domestication and modification of the pOPIN vectors to generate the pOPIN-GG vectors

194 We used the pOPIN-F vector (Berrow et al., 2007) as template to generate Golden Gate 195 compatible versions of the pOPIN vectors, making considerable effort to minimise alterations 196 to the existing pOPIN-F vector backbone. To adapt pOPIN-F to the Golden Gate cloning 197 system, we removed all of the native Type IIS Bsal and Bpil restriction sites from the sequence, a process traditionally termed 'domestication'. After domestication, we generated two different antibiotic resistance variants (carbenicillin and kanamycin) conducive to the need

200 for dual protein co-expression. We then took the carbenicillin resistant $\left(\right.$ Carb $\left.^{R}\right)$ and kanamycin resistant $\left(\operatorname{Kan}^{R}\right)$ vectors and engineered two variants for each to allow for the insertion GOls compatible with either $\mathrm{N}$-terminal or C-terminal tags. To do this, we reintroduced two Bsal sites between the T7 promoter and terminator, which would yield 5' CCAT and 3' GCTT nucleotide overhangs for the N-terminal tag compatible vectors, and 5' AATG and 3' GCTT overhangs in the C-terminal tag compatible vectors post treatment with Bsal (Figure 1). Ultimately, we developed four pOPIN-GG acceptor vectors, pPGN-C (Carb ${ }^{R}$ ) and pPGN-K $\left(\mathrm{Kan}^{R}\right)$ for N-terminal tagging, and pPGC-C $\left(\mathrm{Carb}^{R}\right)$ and pPGC-K $\left(\operatorname{Kan}^{R}\right)$ for C-terminal tagging (Table 1). Further, to assist with positive clone identification, we introduced a visible red fluorescent protein (RFP) negative selection marker, allowing users to select positive white colonies after transformation. Importantly, the $\mathrm{Carb}^{R}$ and $\mathrm{Kan}^{R}$ versions of the acceptors also contain different origins of replication to allow for efficient co-expression in addition to cotransformation.

To overcome complications with PCR (polymerase read-through in the native pOPIN-F vector), we removed a 15xG homopolymer string by excising a 947bp (Eagl-Sacll) section of vector backbone using traditional restriction enzymes and stored this for later use. As a consequence of increased Golden Gate assembly efficiency from cloned (i.e. non-linear)

218 fragments, the remaining sections of the backbone (incorporating changes to the native 219 2xBsal and 2xBpil restriction sites) were amplified by PCR and cloned into custom level 0 
acceptors. Once independently sub-cloned, the mutated fragments could subsequently be reassembled (along with the Eagl-Sacll cassette), eliminating all the existing Bsal and Bpil restriction sites to generate a fully domesticated version of the original pOPIN-F vector.

224 Following domestication, larger sections of the vector back-bone could be re-cloned into additional custom level 0 acceptors to enable a pre-domesticated iGEM RFP negative selection reporter cassette (originating from Discosoma striata) to be inserted in place of the pOPIN-F N-terminal 6xHIS tag and Lac operon (LacZ) negative selection elements. The border sequences immediately flanking this region were found to be important for protein expression and were modified to directly mimic those of the native pOPIN vectors. Final vector assembly was achieved by ligating vector back-bone fragments together along with the EaglSacll and RFP negative selection reporter elements. Changes to the Golden Gate cloning insert overhangs, and exchange of the vector bacterial antibiotic resistance, were achieved by making the necessary modifications to the respective level 0 assembly components.

Cloning of AVR-PikF into pOPIN and pOPIN-GG vectors for test expression and purification

The AVR-PikF coding sequence (encoding residues starting from the end of the signal peptide 21 - 113) was cloned into pOPIN and pOPIN-GG vectors via Infusion cloning and Golden Gate, respectively. Infusion cloning of AVR-PikF in the pOPIN vectors, pOPIN-F, pOPIN-S3C, pOPIN-M and pOPIN-E was performed as described by (Berrow et al., 2007). To clone AVRPikF into the pOPIN-GG constructs, the AVR-PikF sequence was amplified with primers that introduced overhangs at the 5' and 3' of the sequence containing a Bsal Type IIS endonuclease sites (Table 1) which would reveal 5' CCAT and 3' GCTT overhangs or 5' AATG and 3' TTCG overhangs, for N-terminal tagged or C-terminal tagged constructs, respectively

244 (Figure 1). AVR-PikF amplicons were subsequently used in a one-pot Golden Gate reaction 245 (Engler et al., 2009, 2008) with the pPGN-C acceptor for N-terminal tagging or the pPGC-C 246 for C-terminal tagging and desired N-/C-terminal tag-containing construct (Figure 1). Golden

247 Gate reactions were then transformed into chemicompetent Stellar E. coli cells (Takara Bio) 
and positive clones were identified via RFP selection before sequencing to confirm the cloning was successful.

250

251 Small Scale Expression and Purification of differentially tagged AVR-PikF in pOPIN and pOPIN-GG vectors

Constructs for protein expression were transformed into E. coli SHuffle cells (NEB) via heat shock. Protein expression was performed via autoinduction (Studier, 2005) under the required selection. Cells were harvested by centrifugation and resuspended in lysis buffer $(50 \mathrm{mM}$ HEPES pH 7.4, $500 \mathrm{mM} \mathrm{NaCl}, 30 \mathrm{mM}$ imidazole, $5 \mathrm{mM}$ glycine, $5 \%$ glycerol) at a ratio of $5: 1$ buffer per gram of cell pellet. $8 \mathrm{~mL}$ of resuspended cells were transferred to a 24 well plate and lysed by sonication with a 24-tip sonication horn. Cell lysate was clarified by centrifugation at $45,000 \mathrm{~g}$ for 20 mins. $500 \mu \mathrm{l}$ of Ni-NTA resin (Qiagen) was added to each of the clarified cell lysates and incubated for 20 mins with gentle shaking. After incubation, cell lysates with Ni-NTA resin were transferred to $15 \mathrm{ml}$ gravity flow columns and Ni-NTA resin was washed with 2 column volumes of lysis buffer to remove non-specific interactors. Proteins were eluted from the Ni-NTA resin with $1 \mathrm{~mL}$ of elution buffer $(50 \mathrm{mM}$ HEPES pH 7.4, $500 \mathrm{mM} \mathrm{NaCl}, 500$ $\mathrm{mM}$ imidazole). Samples were visualised by SDS-PAGE with $16 \%$ Teo-tricine polyacrylamide gels (Abcam) stained with Instant Blue ${ }^{\circledR}$ Coomassie Stain (Abcam).

Expression and purification of the AVR-PikF and OsHIPP19 complex

A 6xHIS-GB1-tagged OsHIPP19 in pPGN-C was co-transformed with an untagged AVR-PikF in pPGC-K into E. coli SHuffle cells and plated on carbenicillin + kanamycin LB agar plates. Protein expression was performed via autoinduction (Studier, 2005) under carbenicillin + kanamycin selection. Cells were harvested by centrifugation and resuspended in lysis buffer

272 (50 mM HEPES pH 7.4, $500 \mathrm{mM} \mathrm{NaCl}, 30 \mathrm{mM}$ imidazole, $5 \mathrm{mM}$ glycine, $5 \%$ glycerol) at a ratio of $5: 1$ buffer per gram of cell pellet. Cells were lysed via sonication and lysate was clarified by centrifugation at $45,000 \mathrm{~g}$. Clarified lysate was then subjected to Nickel immobilised metal affinity chromatography (IMAC) followed by size exclusion chromatography (SEC) with a 
bioRxiv preprint doi: https://doi.org/10.1101/2021.08.10.455798; this version posted August 10,2021 . The copyright holder for this preprint

(which was not certified by peer review) is the author/funder, who has granted bioRxiv a license to display the preprint in perpetuity. It is made available under aCC-BY 4.0 International license.

276 Superdex S75 26/600. Fractions from SEC were visualised by SDS-PAGE with $16 \%$ Teo-

277 tricine polyacrylamide gels (Abcam) stained with Instant Blue ${ }^{\circledR}$ Coomassie Stain (Abcam). 


\section{Acknowledgements}

279 We thank Ray Owens from the Oxford Protein Production Facility for provision of the pOPIN

280 vectors. We thank Dr. Florian Altegoer for kindly providing pEMGB1 vector for the cloning of

281 6xHIS-GB1 tag. This work was supported by UKRI-BBSRC (grant BB/P012574), UKRI-

282 BBRSC Norwich Research Park Biosciences Doctoral Training Partnership, (grant

283 BB/M011216/1, for FAV) and the ERC (proposal 743165), the ERAMUS ${ }^{+}$training programme,

284 the John Innes Foundation and the Gatsby Charitable Foundation.

\section{Data Availability Statement}

287 All data supporting the findings of this study are contained within the manuscript. The pOPINGG vectors are available directly through

Addgene (https://www.addgene.org/Mark_Banfield/). 


\section{References}

Bentham, A.R., Petit-Houdenot, Y., Win, J., Chuma, I., Terauchi, R., Banfield, M.J., Kamoun, S., Langner, T., 2021. A single amino acid polymorphism in a conserved effector of the multihost blast fungus pathogen expands host-target binding spectrum. BioRxiv 2021.03.15.435478. https://doi.org/10.1101/2021.03.15.435478

Berrow, N.S., Alderton, D., Sainsbury, S., Nettleship, J., Assenberg, R., Rahman, N., Stuart, D.I., Owens, R.J., 2007. A versatile ligation-independent cloning method suitable for high-throughput expression screening applications. Nucleic Acids Res 35, e45. https://doi.org/10.1093/nar/gkm047

di Guana, C., Lib, P., Riggsa, P.D., Inouyeb, H., 1988. Vectors that facilitate the expression and purification of foreign peptides in Escherichia coli by fusion to maltose-binding protein. Gene 67, 21-30. https://doi.org/10.1016/0378-1119(88)90004-2

Engler, C., Gruetzner, R., Kandzia, R., Marillonnet, S., 2009. Golden Gate Shuffling: A OnePot DNA Shuffling Method Based on Type Ils Restriction Enzymes. PLOS ONE 4, e5553. https://doi.org/10.1371/journal.pone.0005553

Engler, C., Kandzia, R., Marillonnet, S., 2008. A One Pot, One Step, Precision Cloning Method with High Throughput Capability. PLOS ONE 3, e3647. https://doi.org/10.1371/journal.pone.0003647

Engler, C., Youles, M., Gruetzner, R., Ehnert, T.-M., Werner, S., Jones, J.D.G., Patron, N.J., Marillonnet, S., 2014. A Golden Gate Modular Cloning Toolbox for Plants. ACS Synth. Biol. 3, 839-843. https://doi.org/10.1021/sb4001504

Kobashigawa, Y., Kumeta, H., Ogura, K., Inagaki, F., 2009. Attachment of an NMR-invisible solubility enhancement tag using a sortase-mediated protein ligation method. J Biomol NMR 43, 145. https://doi.org/10.1007/s10858-008-9296-5

Longya, A., Chaipanya, C., Franceschetti, M., Maidment, J.H.R., Banfield, M.J., Jantasuriyarat, C., 2019. Gene Duplication and Mutation in the Emergence of a Novel Aggressive Allele of the AVR-Pik Effector in the Rice Blast Fungus. MPMI 32, 740-749. https://doi.org/10.1094/MPMI-09-18-0245-R

Maidment, J.H.R., Franceschetti, M., Maqbool, A., Saitoh, H., Jantasuriyarat, C., Kamoun, S., Terauchi, R., Banfield, M.J., 2021. Multiple variants of the fungal effector AVR-Pik bind the HMA domain of the rice protein OsHIPP19, providing a foundation to engineer plant defense. Journal of Biological Chemistry 296, 100371. https://doi.org/10.1016/j.jbc.2021.100371

Malakhov, M.P., Mattern, M.R., Malakhova, O.A., Drinker, M., Weeks, S.D., Butt, T.R., 2004. SUMO fusions and SUMO-specific protease for efficient expression and purification of proteins. J Struct Func Genom 5, 75-86. https://doi.org/10.1023/B:JSFG.0000029237.70316.52

Neuhold, J., Radakovics, K., Lehner, A., Weissmann, F., Garcia, M.Q., Romero, M.C., Berrow, N.S., Stolt-Bergner, P., 2020. GoldenBac: a simple, highly efficient, and widely applicable system for construction of multi-gene expression vectors for use with the baculovirus expression vector system. BMC Biotechnol 20, 26. https://doi.org/10.1186/s12896-020-00616-z

Outram, M.A., Sung, Y.-C., Yu, D., Dagvadorj, B., Rima, S.A., Jones, D.A., Ericsson, D.J., Sperschneider, J., Solomon, P.S., Kobe, B., Williams, S.J., 2021. The crystal structure of SnTox3 from the necrotrophic fungus Parastagonospora nodorum reveals a unique effector fold and provides insight into Snn3 recognition and prodomain protease processing of fungal effectors. New Phytologist n/a. https://doi.org/10.1111/nph.17516

Padgett, K.A., Sorge, J.A., 1996. Creating seamless junctions independent of restriction sites in PCR cloning. Gene 168, 31-35. https://doi.org/10.1016/0378-1119(95)007318

Patron, N.J., Orzaez, D., Marillonnet, S., Warzecha, H., Matthewman, C., Youles, M., Raitskin, O., Leveau, A., Farré, G., Rogers, C., Smith, A., Hibberd, J., Webb, A.A.R., Locke, J., Schornack, S., Ajioka, J., Baulcombe, D.C., Zipfel, C., Kamoun, S., Jones, 
J.D.G., Kuhn, H., Robatzek, S., Esse, H.P.V., Sanders, D., Oldroyd, G., Martin, C., Field, R., O'Connor, S., Fox, S., Wulff, B., Miller, B., Breakspear, A., Radhakrishnan, G., Delaux, P.-M., Loqué, D., Granell, A., Tissier, A., Shih, P., Brutnell, T.P., Quick, W.P., Rischer, H., Fraser, P.D., Aharoni, A., Raines, C., South, P.F., Ané, J.-M., Hamberger, B.R., Langdale, J., Stougaard, J., Bouwmeester, H., Udvardi, M., Murray, J.A.H., Ntoukakis, V., Schäfer, P., Denby, K., Edwards, K.J., Osbourn, A., Haseloff, J., 2015. Standards for plant synthetic biology: a common syntax for exchange of DNA parts. New Phytologist 208, 13-19.

https://doi.org/10.1111/nph.13532

Rosano, G.L., Ceccarelli, E.A., 2014. Recombinant protein expression in Escherichia coli: advances and challenges. Front. Microbiol. 5. https://doi.org/10.3389/fmicb.2014.00172

Studier, F.W., 2005. Protein production by auto-induction in high-density shaking cultures. Protein Expression and Purification 41, 207-234. https://doi.org/10.1016/j.pep.2005.01.016

Zhou, P., Wagner, G., 2010. Overcoming the Solubility Limit with Solubility-Enhancement Tags: Successful Applications in Biomolecular NMR Studies. J Biomol NMR 46, $23-$ 31. https://doi.org/10.1007/s10858-009-9371-6 

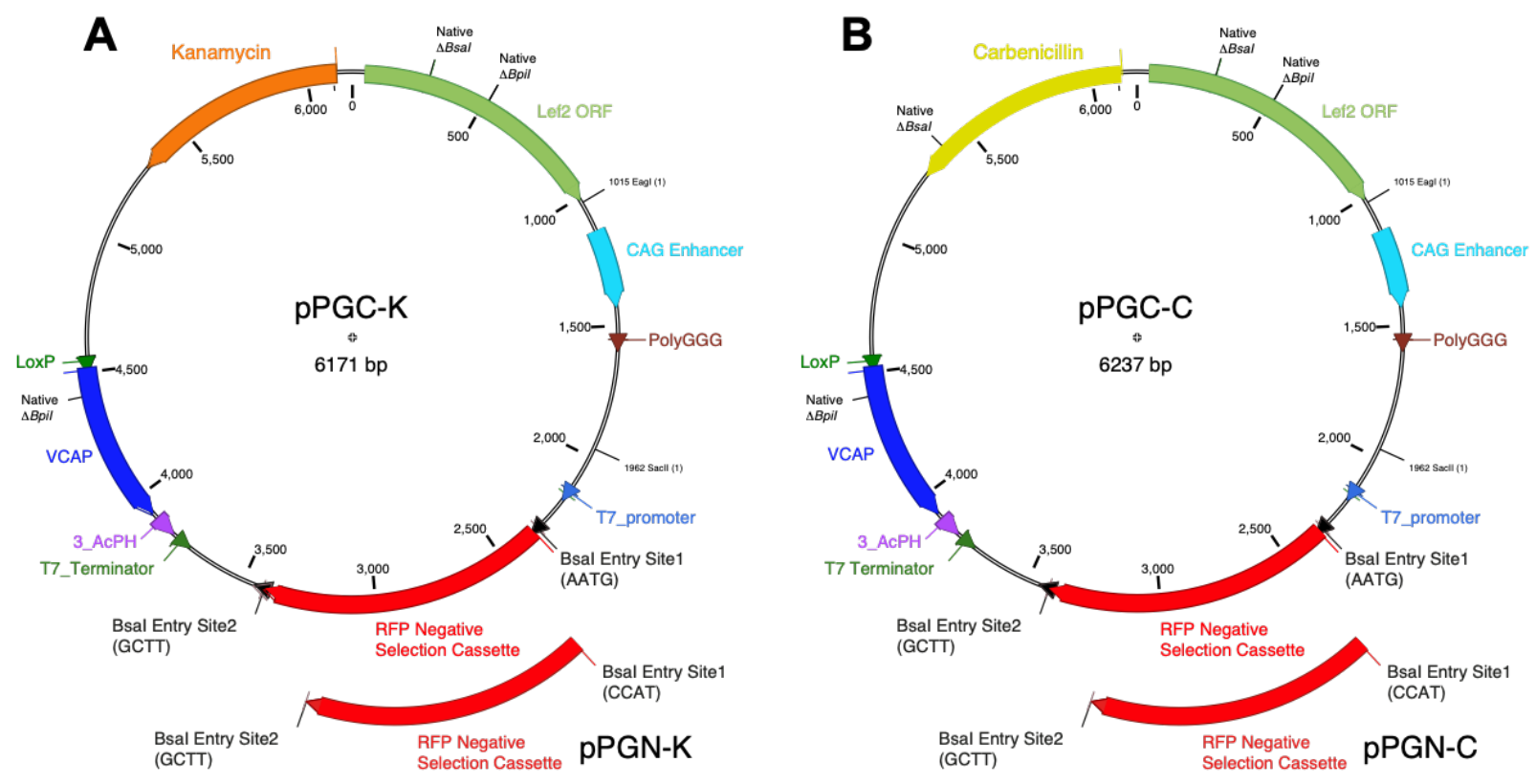

363 Figure 1. Annotated vector maps of the pOPIN-GG acceptors. pOPIN vectors pOPIN-A

364 and pOPIN-F were modified to generate four pOPIN-GG acceptors. A) pPGN-K and pPGC-K are $\operatorname{Kan}^{R}$ vectors descended from pOPIN-A for N-terminal and C-terminal tagging, respectively. B) pPGN-C and pPGC-C are $\mathrm{Carb}^{R}$ vectors descended from pOPIN-F for N-

367 terminal and C-terminal tagging, respectively. $\Delta B$ sal and $\Delta B$ pil represent domesticated Bsal 368 and Bpil sites to allow for Golden Gate cloning compatibility. An RFP negative selection 369 cassette was integrated into the backbone between the Bsal entry sites to allow for red/white selection upon insertion of the GOI. 


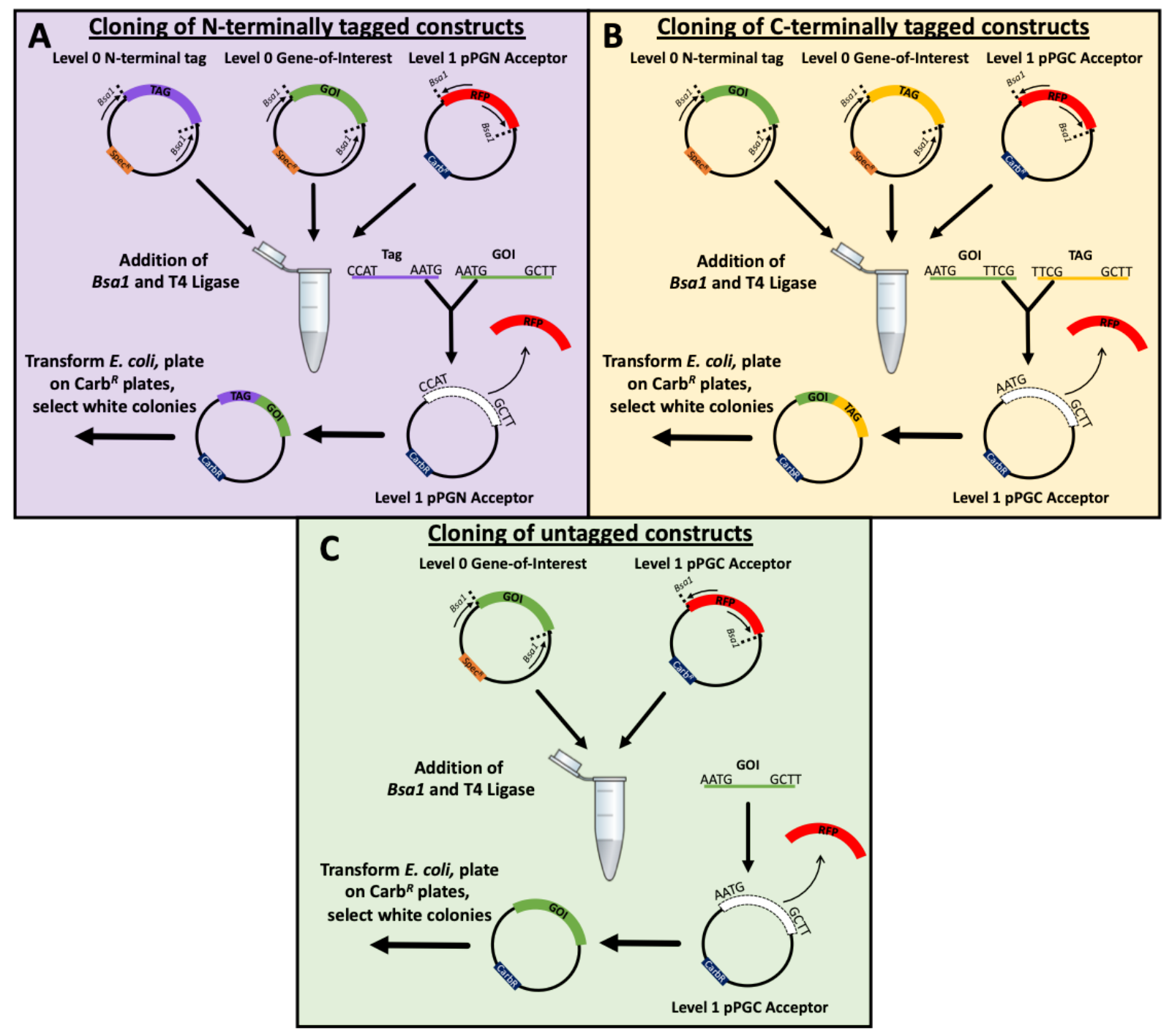

371 Figure 2. Cloning strategy flowcharts. The pOPIN-GG vectors enable N-terminally tagged,

372 C-terminally tagged, or untagged constructs. A) Cloning strategy for N-terminal tagging. GOI

373 requires overhangs 5' AATG and 3' GCTT which can be revealed from Bsal treatment of a 374 level 0 vector or added by PCR. The GOI can then been combined with one of the pPGN 375 acceptor and $\mathrm{N}$-terminal tag level 0 vectors to generate an $\mathrm{N}$-terminally tagged level 1 376 construct. B) Cloning strategy for C-terminal tagging. GOI requires overhangs 5' AATG and 3'

377 TTCG which can be revealed from Bsal treatment of a level 0 vector or added via PCR. The 378 GOI can then be combined with one of the PPGC acceptor and C-terminal tag level 0 vectors 
379 to generate a C-terminally tagged level 1 construct. C) Cloning strategy for generating an

380 untagged gene. As for N-terminal tagging, the GOI requires overhangs 5' AATG and 3' GCTT

381 which can be revealed from Bsal treatment of a level 0 vector or added by PCR. However, is

382 then combined with one of the PPGC vectors, resulting in an untagged GOI, useful in co-

383 expression. Carb ${ }^{R}$ level 1 acceptor vectors are shown for simplicity, $\operatorname{Kan}^{R}$ level 1 acceptor

384 vectors can be used instead as required. 


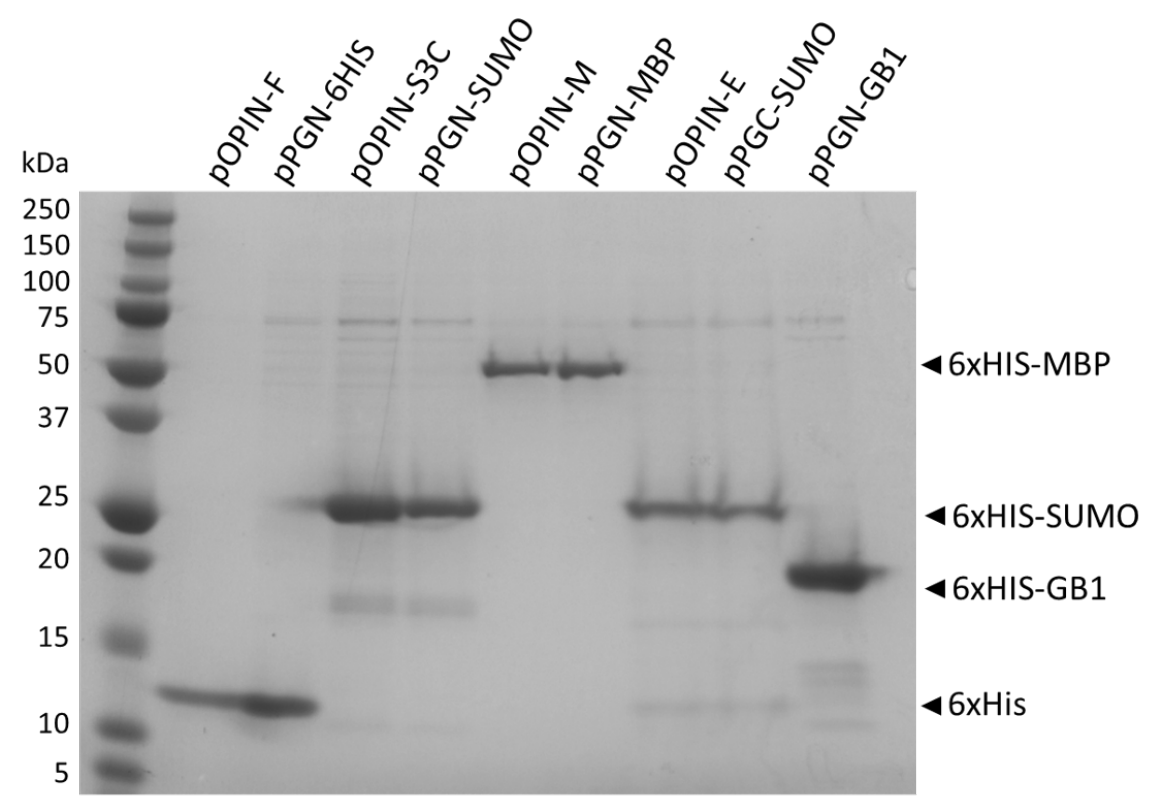

385 Figure 3. Expression and purification of AVR-PikF in pOPIN vectors vs AVR-PikF in pOPIN-GG vectors. AVR-PikF was cloned into the pOPIN vectors pOPIN-F, pOPIN-S3C, pOPIN-M, and pOPIN-E (with additional SUMO tag to aid solubility). The equivalent constructs were produced in the pOPIN-GG system, as well as an additional 6xHIS-GB1-tagged construct. Constructs were expressed in E. coli SHuffle cells and purified using Ni-NTA affinity resin before being visualised by SDS-PAGE. 


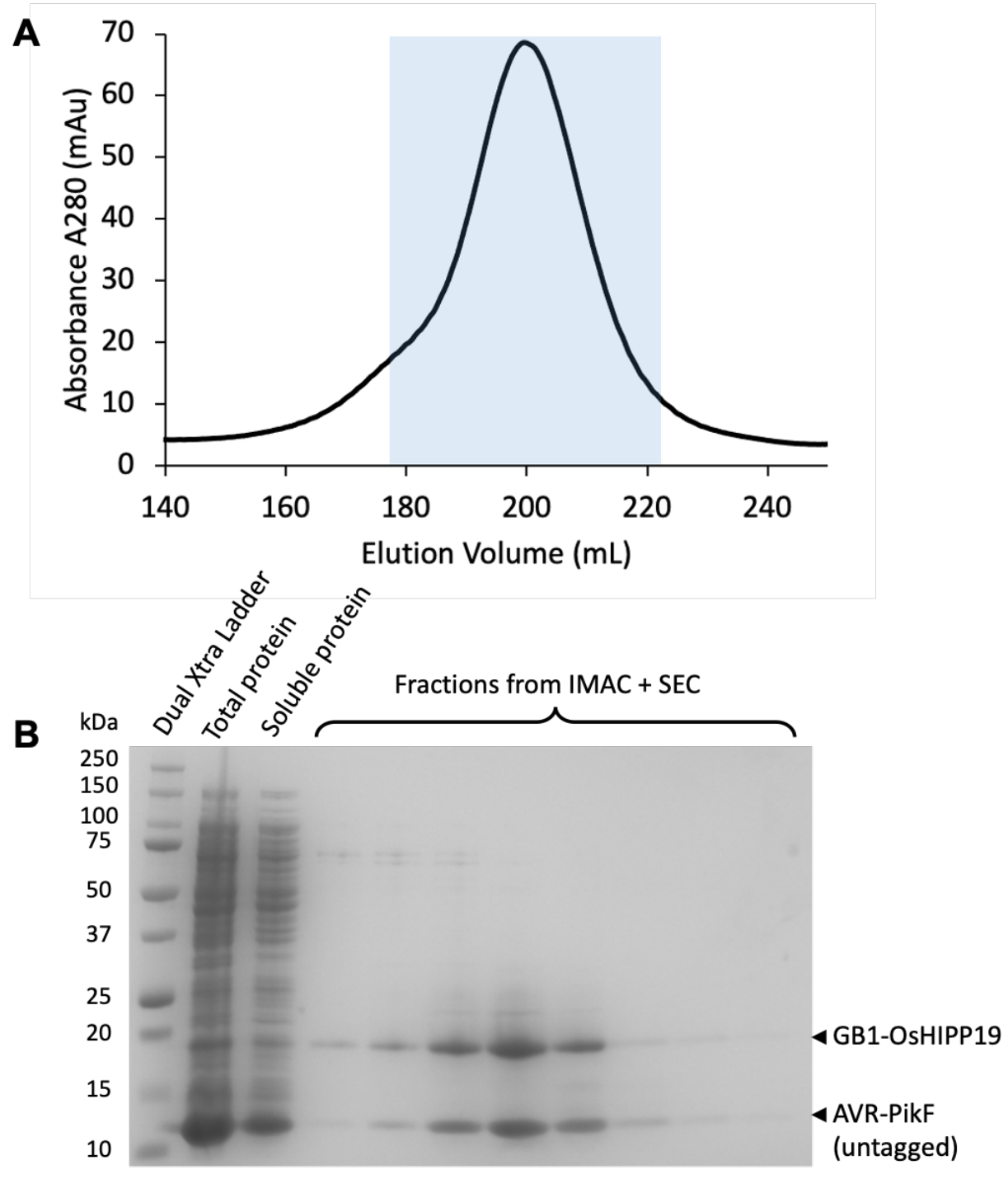
GG system. An untagged construct of AVR-PikF was generated using pPGC-K and coexpressed with OsHIPP19 cloned with a 6xHIS-GB1 solubility tag in pPGN-C E. coli SHuffle cells. Constructs were purified using $\mathrm{Ni}^{2+}$-mediated immobilised metal affinity chromatography (IMAC) coupled with size exclusion chromatography (SEC) before visualised via SDS-PAGE. A) SEC chromatogram of the AVR-PikF / OsHIPP19 complex. Blue box indicates the fractions analysed by SDS-PAGE. B) Instant blue ${ }^{\circledR}$ Coomassie-stained SDS-PAGE gel of the fractions from SEC showing the co-elution of the AVR-PikF and OsHIPP19 proteins, indicating complex formation. 
400 Table 1. Overhang sequences for primers to clone tags and inserts into various

401 acceptors.

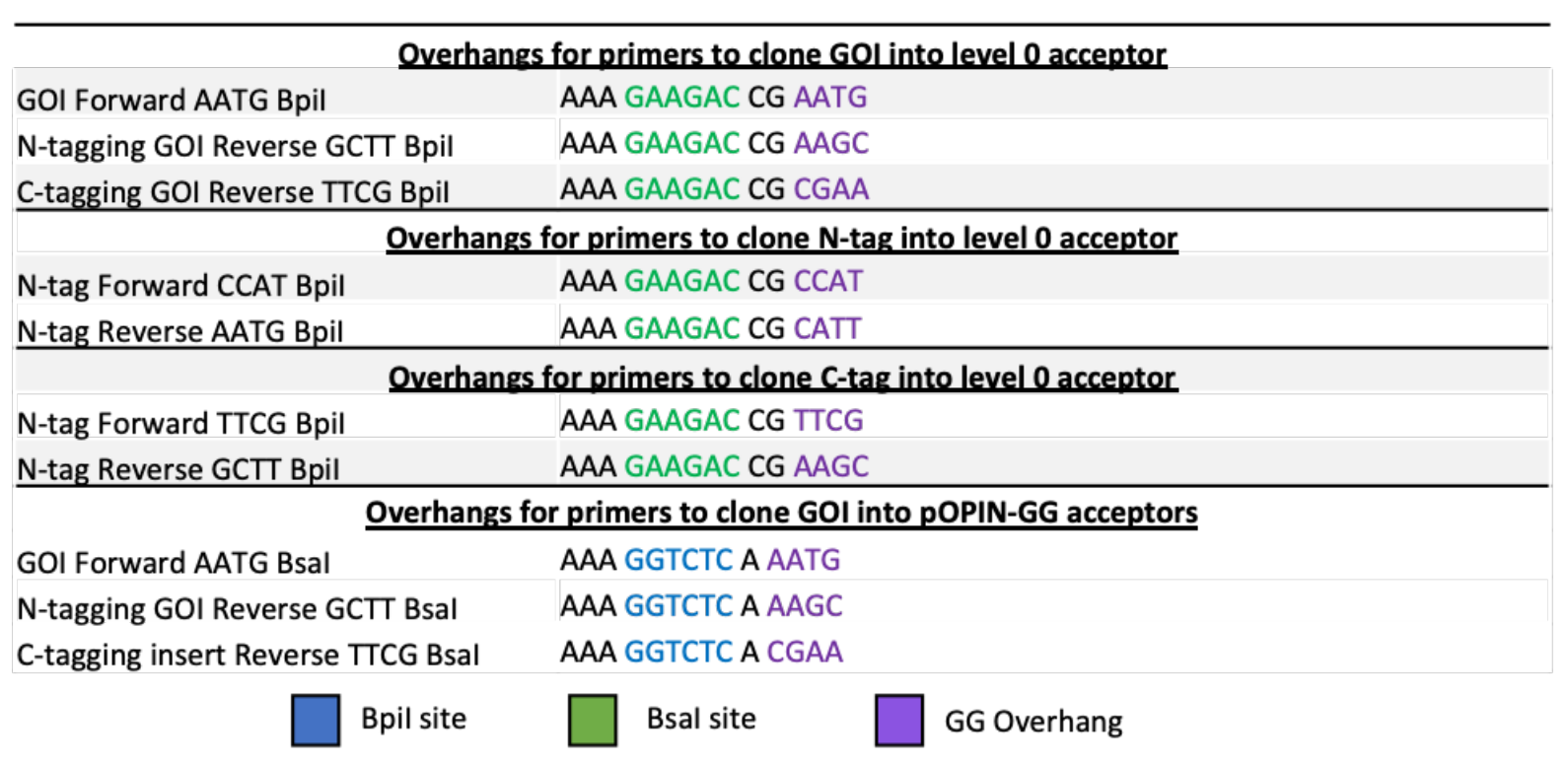


402 Table 2. pOPIN-GG acceptors and compatible vectors available on Addgene

\begin{tabular}{|c|c|c|c|c|c|}
\hline Vectors & Description & 5' Overhang & 3' Overhang & Selection & Addgene ID \\
\hline \multicolumn{6}{|c|}{ Level 0 acceptors for making level 0 modules } \\
\hline $\mathrm{plCH} 41308$ & Acceptor for CDS to be $\mathrm{N}$-tagged & AATG & GCTT & Spectinomycin & 174574 \\
\hline pICSL01005 & Acceptor for CDS to be C-tagged & AATG & TTCG & Spectinomycin & 174575 \\
\hline pICSL01002 & $\begin{array}{l}\text { Acceptor for creating } \mathrm{N} \text {-tag } \\
\text { modules }\end{array}$ & CCAT & AATG & Spectinomycin & 174576 \\
\hline plCSL01003 & $\begin{array}{l}\text { Acceptor for creating C-tag } \\
\text { modules }\end{array}$ & TTCG & GCTT & Spectinomycin & 174577 \\
\hline \multicolumn{6}{|c|}{ Level 1 acceptors for protein expression } \\
\hline pPGN-C & $\begin{array}{l}\text { Expression vector compatible with } \\
\mathrm{N} \text {-terminal tagging }\end{array}$ & CCAT & GCTT & Carbenicillin/Ampicillin & 174578 \\
\hline pPGN-K & $\begin{array}{l}\text { Expression vector compatible with } \\
\mathrm{N} \text {-terminal tagging }\end{array}$ & CCAT & GCTT & Kanamycin & 174579 \\
\hline pPGC-C & $\begin{array}{l}\text { Expression vector compatible with } \\
\text { C-terminal tagging }\end{array}$ & AATG & GCTT & Carbenicillin/Ampicillin & 174580 \\
\hline pPGC-K & $\begin{array}{c}\text { Expression vector compatible with } \\
\text { C-terminal tagging }\end{array}$ & AATG & GCTT & Kanamycin & 174581 \\
\hline \multicolumn{6}{|c|}{ Premade level 0 modules encoding $\mathrm{N}$-terminal tags } \\
\hline pICSL30015 & 6xHIS-MBP-3C & CCAT & $\overline{A A T G}$ & Spectinomycin & 174582 \\
\hline pICSL30017 & 6xHIS uncleavable & CCAT & AATG & Spectinomycin & 174583 \\
\hline plCSL30018 & $6 x H I S-S U M O-3 C$ & CCAT & AATG & Spectinomycin & 174584 \\
\hline pICSL30019 & $6 \mathrm{xHIS}-3 \mathrm{C}$ & CCAT & AATG & Spectinomycin & 174585 \\
\hline pICSL30022 & Twin Streptavidin uncleavable & CCAT & AATG & Spectinomycin & 174586 \\
\hline plCSL30028 & $6 \mathrm{xHIS-GB1}$ & CCAT & AATG & Spectinomycin & 174587 \\
\hline \multicolumn{6}{|c|}{ Premade level 0 modules encoding C-terminal tags } \\
\hline pICSL50001 & 6xHIS-TEV-3xFLAG & TTCG & GCTT & Spectinomycin & 174588 \\
\hline pICSL50025 & 6xHIS uncleavable & TTCG & GCTT & Spectinomycin & 174589 \\
\hline pICSL50026 & $3 \mathrm{C}-6 \mathrm{xHIS}$ & TTCG & GCTT & Spectinomycin & 174590 \\
\hline plCSL50027 & Twin Streptavidin uncleavable & TTCG & GCTT & Spectinomycin & 174591 \\
\hline
\end{tabular}

